Supporting Information:

\title{
Hyphenating Centrifugal Partition Chromatography with Nuclear Magnetic Resonance through automated Solid Phase Extraction.
}

Jonathan Bisson ${ }^{\dagger}$, Marion Brunel ${ }^{\dagger}$, Alain Badoc ${ }^{+, \neq}$, Grégory Da Costa ${ }^{+,}$, Tristan Richard ${ }^{+,}$, JeanMichel Mérillon ${ }^{\dagger}$ Pierre Waffo-Téguo*, ${ }^{*,+}$

†Univ. Bordeaux, Faculté des Sciences Pharmaceutiques, ISVV, EA 4577, Enologie, Molécules d'Intérêt

Biologique (GESVAB), F-33882 Villenave-d'Ornon, France

* INRA, ISVV, USC 1366 Enologie, F-33882 Villenave-d'Ornon, France

* Tel.: +33 5575759 55; fax: +33 5575759 52. E-mail: pierre.waffoteguo@u-bordeaux2.fr (P. WaffoTéguo). 


\section{CONTENTS}

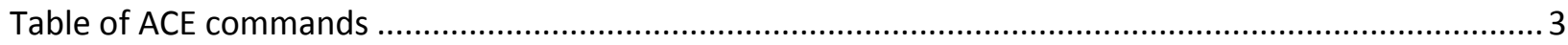

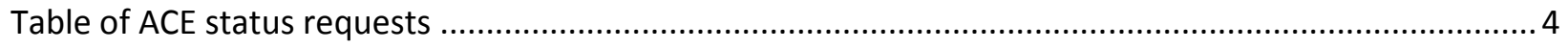

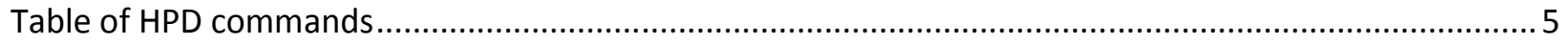

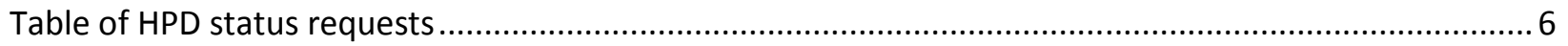

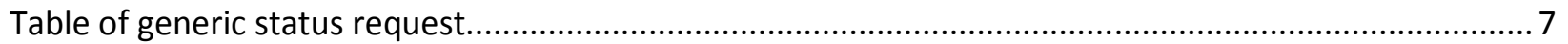

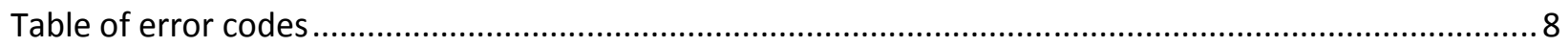

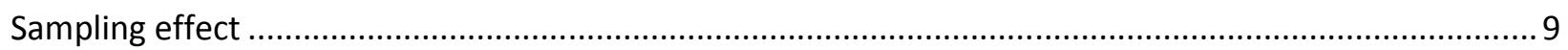

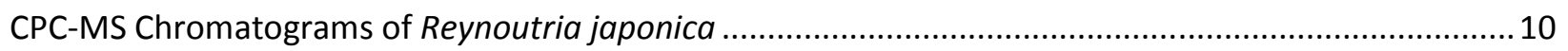

Gnetum africanum, HPLC-UV chromatogram of crude and HLB retention ........................................... 11 
TABLE OF ACE COMMANDS

\begin{tabular}{|r|r|l|l|}
\hline Code & Argument & Function & Long action \\
\hline $\mathbf{1 6 0}$ & 0 & Used during initialization & No \\
\hline $\mathbf{2 1 2 0}$ & 21 & Unload the arm & Yes \\
\hline $\mathbf{2 1 3 7}$ & 1 & Load the arm & Yes \\
\hline $\mathbf{2 1 1 2}$ & $00000 x$ & Change position of valve 1 & Yes \\
\hline $\mathbf{5 1 0 6}$ & 00000x & Change position of valve 2 & Yes \\
\hline $\mathbf{2 1 0 2}$ & 00000x & Change position of valve 3 & Yes \\
\hline $\mathbf{2 1 9 0}$ & 00000x & Change position of valve 4 & Yes \\
\hline $\mathbf{2 1 2 7}$ & $00000 x$ & Change position of the arm (1=left,2=right) & Yes \\
\hline $\mathbf{2 1 1 0}$ & 1 & Initialize left clamp & Yes \\
\hline $\mathbf{2 1 1 0}$ & 0 & Put left clamp in standby position & Yes \\
\hline $\mathbf{2 1 1 1}$ & 1 & Open left clamp & Yes \\
\hline $\mathbf{2 1 1 1}$ & 0 & Close left clamp & Yes \\
\hline $\mathbf{2 1 0 0}$ & 1 & Initialize right clamp & Yes \\
\hline $\mathbf{2 1 0 0}$ & 0 & Put right clamp in standby position & Yes \\
\hline $\mathbf{2 1 0 1}$ & 1 & Open right clamp & Yes \\
\hline $\mathbf{2 1 0 1}$ & 0 & Close right clamp & Yes \\
\hline $\mathbf{2 0 5 0}$ & 1 & Put cartridges back in their position & Yes \\
\hline $\mathbf{2 1 2 2}$ & 0xyyyb & Put cartridge y of rack $x$ in clamp b & Yes \\
\hline $\mathbf{2 1 2 3}$ & 0xyyyb & Put cartridge from clamp b to position y of rack x & Yes \\
\hline $\mathbf{0 1 6 x}$ & $00 y y y y$ & Change output & No \\
\hline
\end{tabular}


TABLE OF ACE STATUS REQUESTS

\begin{tabular}{|r|r|l|}
\hline Code & \multicolumn{1}{|l|}{ Argument } & Function \\
\hline 1001 & 2112 & Valve 1 position \\
\hline 1001 & 5106 & Valve 2 position \\
\hline 1001 & 2102 & Valve 3 position \\
\hline 1001 & 2190 & Valve 4 position \\
\hline 1001 & 2110 & Status of left clamp \\
\hline 1001 & 2100 & Status of right clamp \\
\hline 1001 & 160 & Outputs status \\
\hline 1001 & 169 & Inputs status \\
\hline 1001 & 2130 & RFID code of left rack \\
\hline 1001 & 2133 & RFID code of right rack \\
\hline 1001 & 2132 & RFID type of left rack \\
\hline 1001 & 2135 & RFID type of right rack \\
\hline 1001 & 2172 & RFID status of left rack cartridges \\
\hline 1001 & 2173 & RFID status of right rack cartridges \\
\hline 1001 & 2200 & Cartridge in left clamp \\
\hline 1001 & 2201 & Cartridge in right clamp \\
\hline 1001 & 614 & Valve 1 wear \\
\hline 1001 & 601 & Valve 2 wear \\
\hline 1001 & 612 & Valve 3 wear \\
\hline 1001 & 635 & Valve 4 wear \\
\hline 1001 & 615 & Arm wear \\
\hline 1001 & 2182 & Unknown (answers 0x15) \\
\hline & & \\
\hline 1 & & \\
\hline 1 & &
\end{tabular}




\section{TABLE OF HPD COMMANDS}

\begin{tabular}{|r|r|r|r|}
\hline \multicolumn{1}{|c|}{ Code } & Argument & Function & Long action \\
\hline 2000 & 00000x & Changer position of valve 1 & Yes \\
\hline 2020 & 00000x & Change position of valve 2 & Yes \\
\hline 2001 & 0xxxxx & Draw speed of syringe 1 & No \\
\hline 2005 & 0xxxxx & Pull speed of syringe 1 & No \\
\hline 2021 & 0xxxxx & Draw speed of syringe 2 & No \\
\hline 2025 & 0xxxxx & Pull speed of syringe 2 & No \\
\hline 2002 & 00xxxx & Draw volume x in syringe 1 & Yes \\
\hline 2006 & $00 x x x x$ & Pull volume x from syringe 1 & Yes \\
\hline 2022 & 00xxxx & Draw volume x in syringe 2 & Yes \\
\hline 2026 & 00xxxx & Pull volume x from syringe 2 & Yes \\
\hline 2011 & 000xxx & Pressure limit of syringe 1 & No \\
\hline 2031 & 000xxx & Pressure limit of syringe 2 & No \\
\hline
\end{tabular}


TABLE OF HPD STATUS REQUESTS

\begin{tabular}{|r|r|l|}
\hline \multicolumn{1}{|l|}{ Code } & \multicolumn{1}{|l|}{ Argument } & Function \\
\hline 1001 & 2000 & Valve 1 position \\
\hline 1001 & 2020 & Valve 2 position \\
\hline 1001 & 2010 & Syringe 1 pressure \\
\hline 1001 & 2030 & Syringe 2 pressure \\
\hline 1000 & 2009 & Syringe 1 volume \\
\hline 1000 & 2029 & Syringe 2 volume \\
\hline 1000 & 2001 & Syringe 1 draw speed \\
\hline 1000 & 2005 & Syringe 1 pull speed \\
\hline 1000 & 2021 & Syringe 2 draw speed \\
\hline 1000 & 2025 & Syringe 2 pull speed \\
\hline 1000 & 2011 & Syringe 1 pressure limit \\
\hline 1000 & 2031 & Syringe 2 pressure limit \\
\hline 1001 & 2016 & Syringe 1 current volume \\
\hline 1001 & 2036 & Syringe 2 current volume \\
\hline 1001 & 607 & Valve 1 wear \\
\hline 1001 & 609 & Valve 2 wear \\
\hline
\end{tabular}


TABLE OF GENERIC STATUS REQUEST

\begin{tabular}{|c|c|c|}
\hline Code & Argument & Function \\
\hline 1001 & 152 & Get status of long action \\
\hline 1001 & 154 & Module version \\
\hline 1001 & 155 & Error code 1 \\
\hline 1001 & 156 & Error code 2 \\
\hline 1001 & 158 & Module signature part 1 \\
\hline 1001 & 159 & Module signature part 2 \\
\hline
\end{tabular}




\section{TABLE OF ERROR CODES}

\begin{tabular}{|r|r|l|}
\hline Module & Error code & Error \\
\hline HPD & 300 & Principal controller error \\
\hline HPD & $3 \times 0$ & Syringe controller error $(x=1$ or 2$)$ \\
\hline HPD & $3 \times 1$ & Over-pressure in syringe $(x=1$ or 2$)$ \\
\hline HPD & $3 \times 2,3 \times 3$ & Syringe cannot move $(x=1$ or 2$)$ \\
\hline HPD & $3 \times 8,3 \times 9$ & Syringe controller not working $(x=1$ or 2$)$ \\
\hline ACE & 400 & Principal controller error \\
\hline ACE & 401 & Invalid cartridge \\
\hline ACE & 402 & Non initialized module \\
\hline ACE & 410 & Error in valve 1 or right clamp \\
\hline ACE & 411 & Cartridge was not expected in right clamp \\
\hline ACE & 420 & Error in valve 3 or left clamp \\
\hline ACE & 421 & Cartridge was not expected in left clamp \\
\hline ACE & 440 & Error valve 2 or 4 \\
\hline ACE & 450 & RFID error \\
\hline ACE & $47 x$ & Unknown position \\
\hline ACE & 473 & Solvent tray is not here \\
\hline ACE & 474,487 & No drawer or wrong position of it \\
\hline ACE & 475,476 & Arm cannot move (475 left, 476 right) \\
\hline ACE & $479,481,483$ & A cartridge was expected in the arm \\
\hline ACE & $480,482,484,485$ & A cartridge was not expected in the arm \\
\hline ACE & 488,489 & Error in transporter \\
\hline ACE & 500 & Impossible to start task \\
\hline ACE & 501 & Invalid version \\
\hline ACE & $520,528,529$ & Error in valve controller \\
\hline & & \\
\hline
\end{tabular}


SAMPLING EFFECT - UV TRACE SHOWING THE SPIKES DUE TO THE SAMPLE BEING DIVERTED FROM THE CPC->UV->MS FLOWPATH

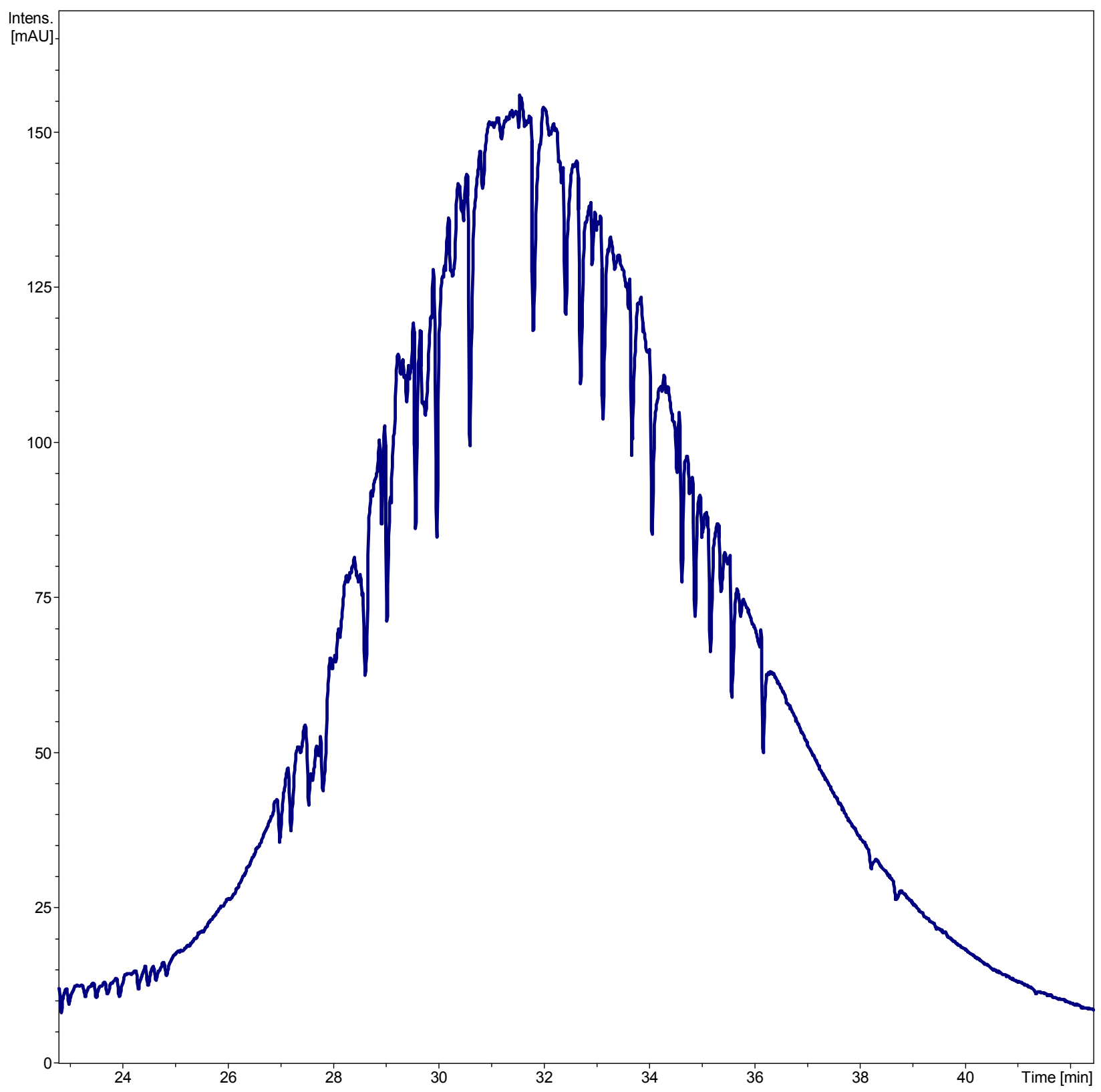




\section{CPC-MS CHROMATOGRAMS OF REYNOUTRIA MULTIFLORA}

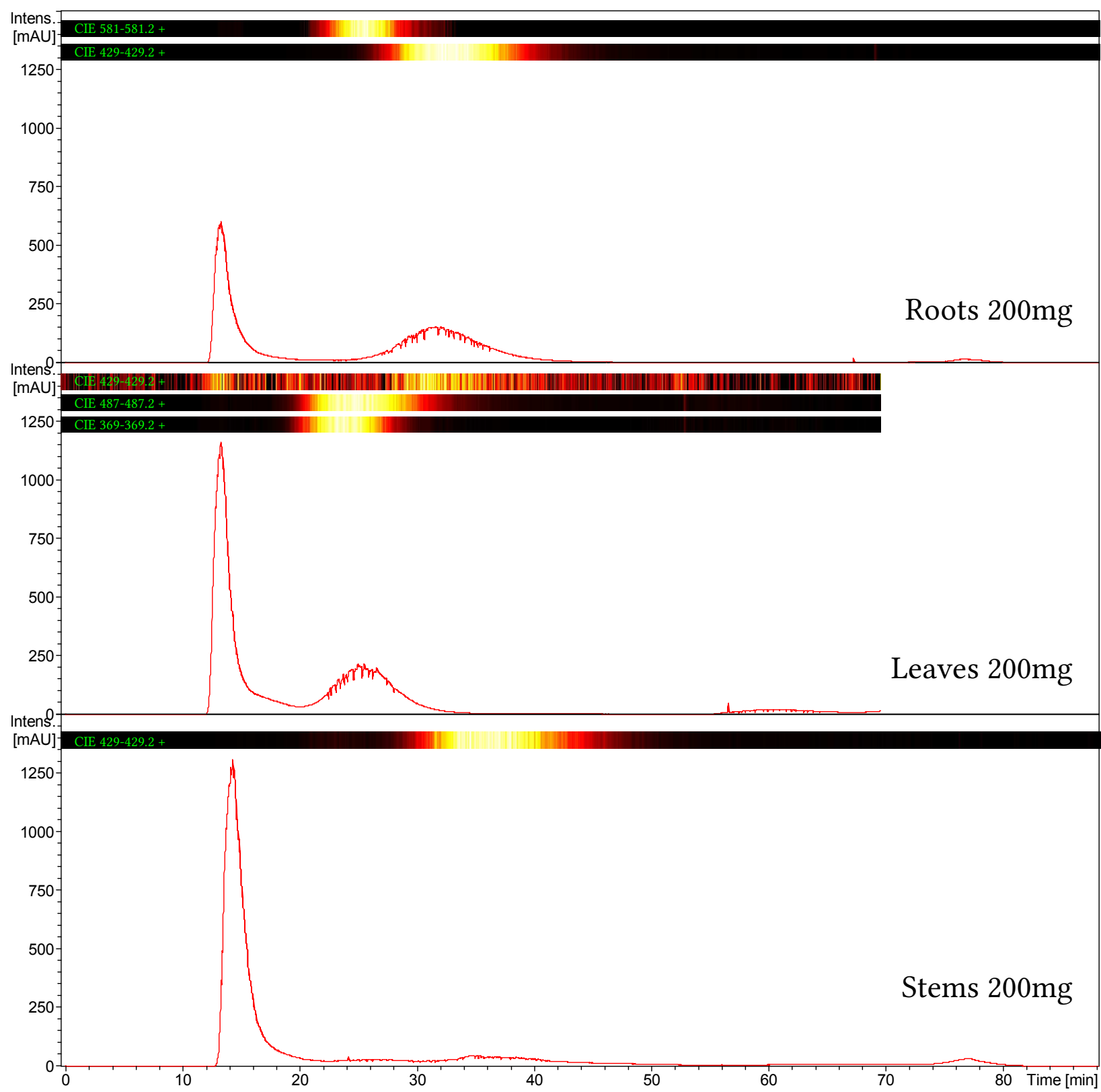




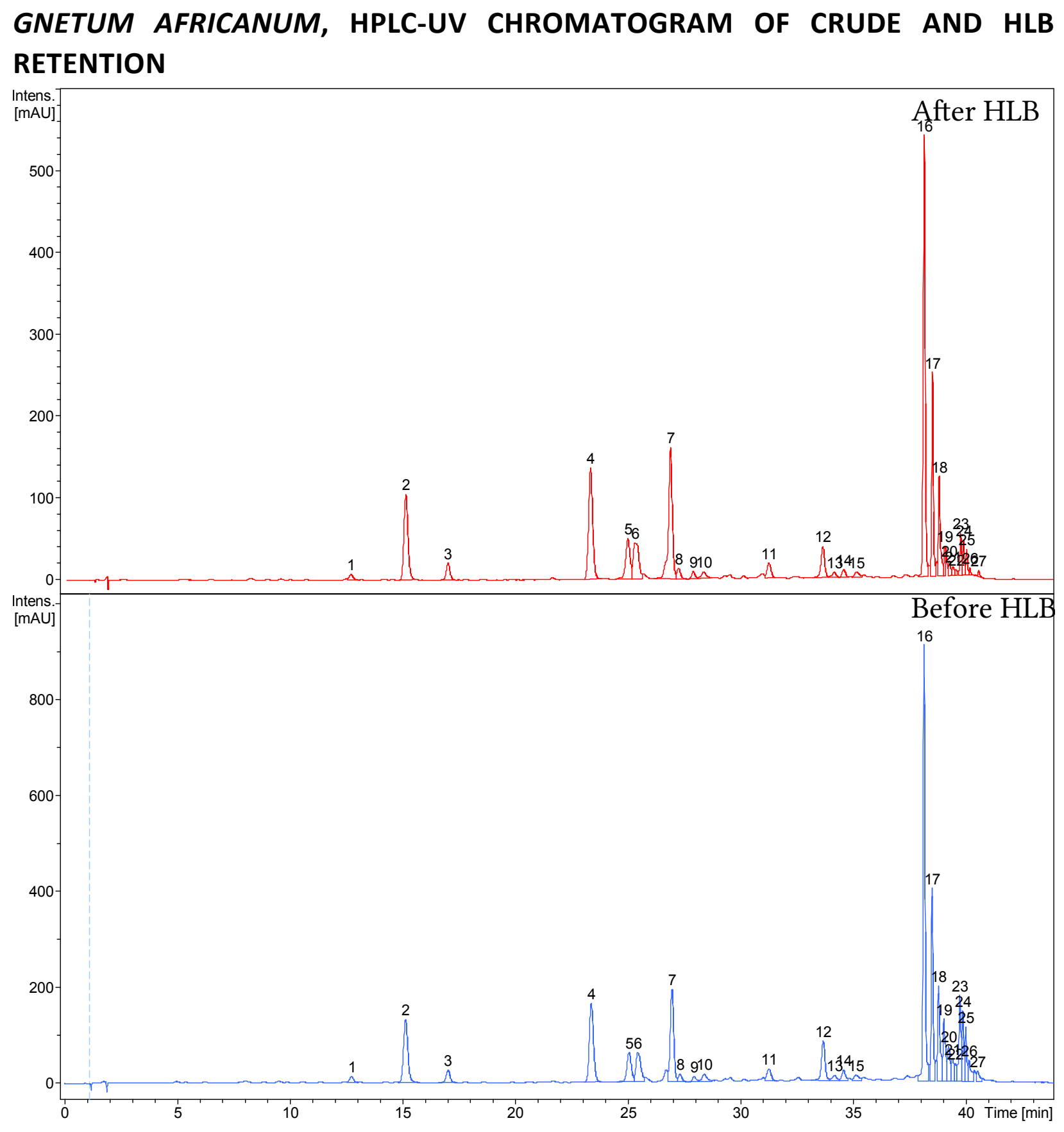

